\title{
The gastrointestinal tolerability of a non-digestible carbohydrate; an ascending dose trial in healthy volunteers
}

\author{
M. Tooley ${ }^{1}$, E.J. Simpson ${ }^{1,2}$ and I.A. Macdonald ${ }^{1,2}$ \\ ${ }^{1}$ University of Nottingham School of Life Sciences, Medical School, Nottingham, NG72UH, UK and \\ ${ }^{2}$ National Institute for Health Research Nottingham Biomedical Research Centre, Queen's Medical Centre, \\ Nottingham, NG7 2UH, UK
}

In 2015, Public Health England challenged food manufacturers to reduce the free sugars content of sweetened food products by $20 \%$, to address the prevalence of obesity within the $\mathrm{UK}^{(1)}$. Whilst progress has been made in reformulating sweetened drinks to reduce their sugar content, the contribution of sucrose to the taste and texture of sweetened snacks and confectionery has been difficult to replace using the readily available non-nutritive sweeteners. $\beta$-linked oligosaccharides (5-9 glucose subunits), derived from acid hydrolysis of cellulose, were identified as a possible replacement for sucrose in these products. The current trial aimed to assess the gastrointestinal (GI) tolerability of consuming these cellodextrins in healthy individuals using a single, ascending 'dose' design.

Thirteen participants (6M:7F; aged 26.5(SD 5.9) years, BMI 24.5(SD 2.4) kg.m ${ }^{-2}$ ) attended the laboratory at 8 am on 4 occasions separated by $\geq 1$ week. On visits $1-4$ respectively, 10, 20, 30, or $50 \mathrm{~g}$ of cellodextrins were consumed in a chocolate-flavoured milkshake (285kJ, $4.0 \mathrm{~g}$ Fat, $4.0 \mathrm{~g}$ Protein, $3.3 \mathrm{~g}$ Carbohydrate). GI symptoms (borborygmus and abdominal discomfort) were assessed before, and every $30 \mathrm{mins}$, for $8 \mathrm{hrs}$ after consuming the drink using visual analogue scales (VAS; 0-100). At 2hrs, a snack bar (Nutri-grain ${ }^{\mathrm{TM}}$, Kelloggs), and at 4 hrs a standard sandwich lunch, were provided. Dietary intake and laxation records (Bristol Stool Score, changes in bowel habits, abdominal discomfort, flatulence) were kept for 3 days prior to, during and for 2 days after each study visit. Participants were withdrawn from the trial if they reported loose stools in the 3-day post-consumption period or abdominal pain $>3$ (out of 5).

One participant was withdrawn after consuming $10 \mathrm{~g}$ due to reporting diarrhoea and abdominal pain on the study day, with one reaching their toleration limit after consuming $30 \mathrm{~g}$. In the remaining 11 participants, no change in mean borborygmus VAS scores, above pre-consumption values, was observed during study days (1-way ANOVA; P $>0.128$ in each case), with no differences in this variable seen between visits (2-way ANOVA; $P=0.380)$. Mean abdominal discomfort scores remained within 10/100 points of preconsumption values throughout the 8hrs (1-way ANOVA; P > 0.319 in each case), and did not differ between visits (2-way ANOVA; $\mathrm{P}=0.491$ ). Across both pre- and post-consumption days, median stool frequency was $1 /$ day (range $0-3 /$ day), with frequency in the post-consumption reporting period being similar between visits (Friedman's test; $\mathrm{P}=0.413$ ). Similarly, total number of stools passed during the post-consumption period were comparable between visits (Friedman's test; $\mathrm{P}=0.913$ ). However, 2 of the 11 participants reported mild abdominal bloating after consuming $30 \mathrm{~g}$, with 7 of 11 reporting bloating and increased flatulence after consuming $50 \mathrm{~g}$ of cellodextrin. It is interesting to note that the participant who was withdrawn after consuming $10 \mathrm{~g}$ had low habitual fibre intake (6g/day) compared to those who completed all 4 visits (19.6 (SD5.3)g/day).

Cellodextrin was tolerated by most participants up to an acute dose of $30 \mathrm{~g}$. However, as with consumption of other indigestible carbohydrates, adverse GI symptoms may be more likely in individuals with low habitual dietary fibre intakes.

This study was funded by the BBSRC through an Innovate UK initiative (BB/L025019/1). Cellodextrins were supplied by Lianhetech Europe.

1. Public Health England (2018) Sugar Reduction Programme. https://publichealthenglandexposureco/sugar-reduction-programme. 